\title{
A percepção da equipe de enfermagem sobre o cuidado à mulher em situação de abortamento: revisão integrativa
}

\author{
The perception of the nursing team on the care of women in a state of abortment: \\ integrative
}

La percepción del equipo de enfermería sobre el cuidado a la mujer en situación de abortación: revisión integrativa

Josiane dos Santos Andrade ${ }^{1}$, Lucas Luzeiro Nonato ${ }^{1}$, Marcio Leles Gama Carneiro ${ }^{1}$, Renilson de Souza Ribeiro ${ }^{1}$, Orácio Carvalho Ribeiro Junior ${ }^{1 *}$.

\section{RESUMO}

Objetivo: Analisar a percepção da equipe de enfermagem sobre o cuidado à mulher em situação de abortamento. Métodos: Trata-se de uma revisão integrativa da literatura, onde foram eleitos 7 artigos nas bases de dados Scientific Electronic Library Online (SCIELO) e Literatura Latino-Americana em Ciências da Saúde (LILACS) no período de janeiro de 2008 a setembro de 2018. Resultados: Os estudos revelam que a percepção dos profissionais de enfermagem sobre a mulher em situação de abortamento é essencialmente negativa, sendo moldada por questões culturais, legais, religiosas e sócio econômicas, fato que gera o preconceito no atendimento a estas mulheres, contribuindo para o mau funcionamento desse serviço. Considerações finais: Ao final percebe-se a percepção evidenciada dos enfermeiros sobre as mulheres em situação de abortamento é extremamente negativa por questões da falta de ética profissional, onde a atitude e valores sociais visam questões religiosas, culturais, econômicas e sociais que influenciam muito negativamente na assistência prestadas a essas pacientes, marginalizando o atendimento devido fazerem julgamentos pessoais.

Palavras-chave: Abortamento, Enfermeiros, Cuidados de enfermagem, Política de saúde, Percepção.

\begin{abstract}
Objective: To analyze the perception of the nursing team about the care of women in abortion situations. Methods: This is an integrative review of the literature, in which 7 articles were selected in the Scientific Electronic Library Online (SCIELO) and Latin American Literature in Health Sciences (LILACS) databases from January 2008 to September 2018 Results: Studies show that the perception of nursing professionals about women in abortion is essentially negative, being shaped by cultural, legal, religious and socioeconomic issues, a fact that generates prejudice in the care of these women, contributing to the malfunction of this service. Final considerations: At the end of the study, the nurses' perceptions about women in abortion are extremely negative due to lack of professional ethics, where social attitudes and values aim at religious, cultural, economic and social issues that have a very negative influence on the assistance to these patients, marginalizing care due to making personal judgments.
\end{abstract}

Keywords: Abortion, Nurses, Nursing care, Health policy, Perception.

${ }^{1}$ Centro Universitário do Norte (UNINORTE), Manaus- AM. *E-mail: o_ra_cio13@hotmail.com SUBMETIDO EM: 3/2019 ACEITO EM: 5/2019 PUBLICADO EM: 10/2020 


\section{RESUMEN}

Objetivo: Analizar la percepción del equipo de enfermería sobre el cuidado a la mujer en situación de aborto. Métodos: Se trata de una revisión integrativa de la literatura, donde fueron elegidos 7 artículos en las bases de datos Scientific Electronic Library Online (SCIELO) y Literatura Latinoamericana en Ciencias de la Salud (LILACS) en el período de enero de 2008 a septiembre de 2018. Resultados: Que la percepción de los profesionales de enfermería sobre la mujer en situación de aborto es esencialmente negativa, siendo moldeada por cuestiones culturales, legales, religiosas y socioeconómicas, hecho que genera el preconcepto en la atención a estas mujeres, contribuyendo al mal funcionamiento de este servicio. Consideraciones finales: Al final se percibe la percepción evidenciada de los enfermeros sobre las mujeres en situación de aborto es extremadamente negativa por cuestiones de la falta de ética profesional, donde la actitud y valores sociales apunta a cuestiones religiosas, culturales, económicas y sociales que influencian muy negativamente en la sociedad la asistencia prestada a esas pacientes, marginando la atención debido a hacer juicios personales.

Palabras clave: Aborto, Enfermeros, Cuidados de enfermería, Política de salud, Percepción.

\section{INTRODUÇÃO}

O produto da concepção eliminado no processo de abortamento é chamado aborto. $\mathrm{O}$ abortamento pode ser precoce, quando ocorre até a $13^{\mathrm{a}}$ semana e tardio quando ocorre entre $13^{\mathrm{a}}$ e $22^{\mathrm{a}}$ semana (BRASIL, 2011). De 210 milhões de gestações que ocorrem a cada ano, metade delas é indesejada, portanto, calcula- se que uma a cada nove dessas mulheres recorre a interrupção, que resulta em cerca de 46 milhões (22\%) de abortamentos indesejados (ROCHA WB, et al., 2015).

No Brasil, segundo o Ministério da Saúde, estima- se que 31\% das gestações terminam em abortamento, corresponde à 1.443 .350 anualmente, o que resulta na taxa de $3,7 \%$ de abortamento para 100 mulheres de 15 a 49 anos, configurando como grande problema de saúde pública relacionado às condições de vida de mulheres (BRASIL, 2005).

Nesse sentido Domingos SRF e Merighi MAB (2010), apontam alguns aspectos que englobam a situação de abortamento, tais como aspectos constitucionais, culturais, religiosos, econômicos, sociais e morais, que por sua vez acabam influenciando no aumento da prevalência de aborto em hospitais clandestinos, principalmente mulheres pobres e de áreas marginalizadas.

Em 1999, o Brasil previu a regulamentação do aborto, ao apresentar as normas técnicas de prevenção e tratamento dos agravos resultantes da violência sexual contra mulheres e a adolescente, atualmente essas normas foram atualizadas, isentando a mulher de apresentar o BO (boletim de ocorrência) ou até mesmo laudo do IML (Instituto Médico Legal) (BRASIL, 1999).

Nota-se que o acesso à saúde, resolutividade dos sinais e sintomas e integralidade são princípios ainda negligenciados quando ocorre um processo de abortamento (BRASIL, 2009). Um estudo realizado em 2007 com médicos ginecologistas e obstetras, apontou que $2 / 3$ dos médicos acreditavam que a autorização judicial era necessária para a realização do aborto previsto por lei (GALLI B, 2010).

O abortamento no Brasil é um assunto que envolve grandes complexidades, questionamentos e muita polêmica, seja nos aspectos legais, bioéticas e ou religiosos e que apesar de muito recorrente no dia a dia do profissional de enfermagem que atua no sistema público de saúde, ainda é um assunto pouco explorado no que diz respeito ao atendimento (DOMINGOS SRF e MERIGHI MAB, 2010).

Nesse sentido é frequente que muitas mulheres experimentam um sentimento de revolta somado ao receio do julgamento e o comportamento dos profissionais de saúde, o que dificulta a boa relação entre profissionais e pacientes, postergando atendimento e por vez, o descaso (ROCHA WB, et al., 2015). 
Para Silva JPL e Araújo MZ (2011), o profissional de saúde, em especial o enfermeiro, necessita estar capacitado para prestar assistência humanizada às mulheres no processo de abortamento e pós-aborto, sendo necessário que os enfermeiros conheçam as alterações físicas e emocionais dessas pacientes, e repensem suas práticas para que suas opiniões pessoais não influenciem na garantia do atendimento humanizado as mulheres.

Após experiências vivenciadas em campo foi possível verificar o déficit na assistência de enfermagem no acolhimento e atendimento de paciente em situação de abortamento. A partir deste olhar sobre aspectos relevantes em relação as situações de abortamento, tem-se como questão o problema desse estudo: como os profissionais de enfermagem percebem o atendimento prestado a mulher no processo de abortamento?

Assim, essa pesquisa tem por objetivo analisar a percepção da equipe de enfermagem sobre o cuidado à mulher em situação de abortamento.

\section{MÉTODOS}

O presente estudo trata-se de uma revisão integrativa da literatura, na qual visa por sua vez analisar e sistematizar resultados de estudos. Segundo Mendes KDS, et al., (2008) a revisão integrativa da literatura dedica-se a forma de contextualizar e investigar evidências sobre determinada questão, de forma ordenada e sistemática, com vistas ao aprofundamento de determinado assunto.

$1^{\underline{a}}$ Fase: elaboração da pergunta norteadora. A presente pesquisa é de natureza teórico-bibliográfica de caráter exploratório com busca em conhecimentos específicos sobre o assunto abordado, nas referências de documentos e autores, predominantemente. Possui a seguinte pergunta norteadora: como os profissionais de enfermagem percebem o atendimento prestado a mulher no processo de abortamento?

$2^{\mathrm{a}}$ Fase: busca ou amostragem na literatura: trabalho. Foram utilizadas referências teóricas por meio de periódicos, artigos científicos, localizados em sites especializados nas bases de dados Scientific Electronic Library Online (SCIELO) e Literatura Latino-Americana em Ciências da Saúde (LILACS).

Foram utilizados para a busca dos artigos os seguintes descritores: "Abortamento", "Enfermeiros", "Cuidados de Enfermagem", "Política de saúde". Os quais foram extraídos do DeCs - Descritores em Ciência da Saúde. A busca dos artigos na base de dados SCIELO processou-se através dos descritores "abortamento", "percepção". Na base de dados LILACS utilizou-se os seguintes descritores: "abortamento", "cuidados de enfermagem".

Quanto aos critérios de inclusão foram selecionados os artigos que estavam em texto completo, publicados no Brasil, em idioma português, que compreendiam o período proposto de janeiro de 2008 a setembro de 2018 e concernentes ao objetivo da revisão. Os critérios de exclusão compreenderam os artigos do tipo revisão de literatura, relatos de experiência, estudos de caso, teses, dissertações, monografias e outras publicações da literatura cinza.

$3^{\text {a }}$ Fase: coleta de dados: Nesta etapa procedeu-se à extração das informações essenciais dos artigos da amostra final da revisão, a partir de um quadro síntese contendo os seguintes dados: Base, Revista, Título, Autor (es), Objetivo, Metodologia e Ano.

4ํㅡㄹ Fase: análise crítica dos estudos incluídos: realizou-se uma leitura minuciosa dos artigos para a busca dos quais abordavam sobre o objetivo do trabalho.

5aㅡ Fase: Nesta etapa os achados da amostra da revisão foram analisados em profundidade, afim de que pudessem ser dissertados para responder a questão inicialmente levantada e, desse modo, procedendo à discussão entre os autores, evidenciando convergências e divergências.

6몰 Fase: apresentação da revisão integrativa: os resultados foram apresentados através do fluxograma e dos quadros. 


\section{RESULTADOS}

Nesta pesquisa foram identificados um total de 40 artigos nas bases: Literatura Latino-Americana em Ciências da Saúde (LILACS) e Scientific Electronic Library Online (SCIELO) no período de janeiro de 2008 a setembro de 2018, sendo que após leitura e revisão foram selecionados 07 artigos como amostra final da revisão, conforme evidenciado na Figura 1.

Figura 1 - Fluxograma de seleção dos artigos para a composição da revisão integrativa.

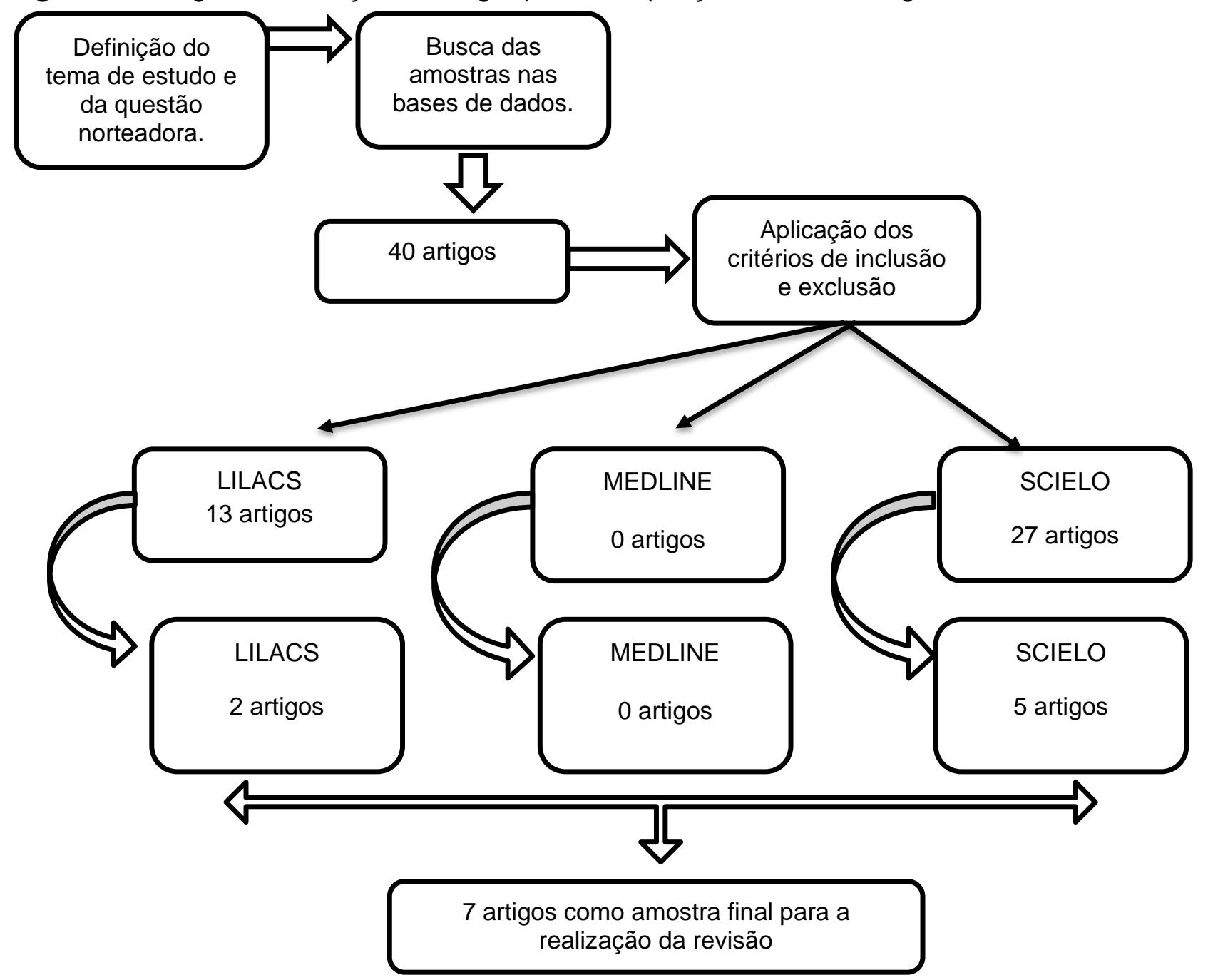

Fonte: Andrade JS, et al., 2020.

Contudo, nota-se uma prevalência maior de publicações na base de dados SCIELO e LILACS, evidenciando a importância desta base para as publicações brasileiras. Por outro lado, observa-se que a maioria das publicações estão em outras revistas de saúde que não as de enfermagem, mostrando a fragilidade dos periódicos da profissão em publicar sobre a temática conforme mostra o quadro 1: Caracterização dos estudos a partir dos seguintes tópicos: Base, Revista, Título, Autor (es), Objetivo, Metodologia e Ano.

De uma forma geral, percebe-se escassez de estudos relacionados à temática na literatura nacional, fato que confirma a essencialidade deste tudo, pois, o mesmo poderá servir de base para a discussão no âmbito da educação permanente nos serviços de atenção, para aprimoramento do cuidado em saúde e em enfermagem. 


\section{Revista Eletrônica Acervo Saúde / Electronic Journal Collection Health ISSN 2178-2091}

Quadro 1 - Caracterização dos estudos a partir dos seguintes tópicos: Base, Revista, Título, Autor (es), Objetivo, Metodologia e Ano.

\begin{tabular}{|c|c|c|c|c|c|c|c|c|}
\hline \multirow{2}{*}{ № } & \multirow{2}{*}{ Base } & \multirow{2}{*}{ Revista } & \multirow{2}{*}{ Título } & \multirow{2}{*}{ Autor (es) } & \multirow{2}{*}{ Objetivo } & \multicolumn{2}{|c|}{ Metodologia } & \multirow{2}{*}{ Ano } \\
\hline & & & & & & Tipo de estudo & Abordagem & \\
\hline 1 & LILACS & $\begin{array}{l}\text { Revista da Escola } \\
\text { de Enfermagem } \\
\text { da USP }\end{array}$ & $\begin{array}{l}\text { Representações de } \\
\text { enfermeiras sobe o } \\
\text { cuidado com mulheres } \\
\text { em situação de aborto } \\
\text { inseguro }\end{array}$ & $\begin{array}{l}\text { Mortari CLH, } \\
\text { et al. }\end{array}$ & $\begin{array}{l}\text { Identificar as representações de enfermeiras da } \\
\text { atenção básica a saúde do município de Chapecó } \\
\text { (SC) sobre a prática do cuidado a mulheres em } \\
\text { situação de abortamento/ aborto inseguro. }\end{array}$ & $\begin{array}{l}\text { Exploratório, } \\
\text { descritiva. }\end{array}$ & Qualitativa & 2012 \\
\hline 2 & LILACS & $\begin{array}{l}\text { Revista Brasileira } \\
\text { de Ciências da } \\
\text { Saúde }\end{array}$ & $\begin{array}{l}\text { Olhar reflexivo sobre o } \\
\text { Aborto na visão da } \\
\text { enfermagem a partir de } \\
\text { uma leitura de gênero. }\end{array}$ & $\begin{array}{l}\text { da Silva JPL } \\
\text { e Araújo MZ }\end{array}$ & $\begin{array}{c}\text { Verificar a construção dos profissionais de } \\
\text { enfermagem sobre o aborto e sua influência na } \\
\text { assistência prestada a mulher em processo de } \\
\text { abortamento. }\end{array}$ & $\begin{array}{l}\text { Exploratório, } \\
\text { descritiva. }\end{array}$ & Qualitativa & 2011 \\
\hline 3 & SCIELO & Revista Nursing & $\begin{array}{l}\text { A contextualização do } \\
\text { aborto sob a ótica do } \\
\text { enfermeiro }\end{array}$ & $\begin{array}{l}\text { Ayres R, et } \\
\quad \text { al. }\end{array}$ & $\begin{array}{l}\text { Analisar opinião de enfermeiros do sexo } \\
\text { masculino quanto ao aborto provocado. }\end{array}$ & $\begin{array}{l}\text { Exploratório, } \\
\text { descritiva. }\end{array}$ & Qualitativa & 2018 \\
\hline 4 & SCIELO & $\begin{array}{l}\text { Escola Anna Nery } \\
\text { Revista de } \\
\text { Enfermagem }\end{array}$ & $\begin{array}{l}\text { Cuidado integral e } \\
\text { aconselhamento } \\
\text { reprodutivo à mulher que } \\
\text { abortou: percepções da } \\
\text { enfermagem. }\end{array}$ & $\begin{array}{l}\text { Strefling ISS, } \\
\text { et al. }\end{array}$ & $\begin{array}{c}\text { conhecer a percepção dos profissionais de } \\
\text { enfermagem a respeito do cuidado integral e do } \\
\text { aconselhamento reprodutivo à mulher que } \\
\text { abortou. }\end{array}$ & $\begin{array}{l}\text { exploratório- } \\
\text { descritivo }\end{array}$ & qualitativa & 2012 \\
\hline 5 & SCIELO & Revista Bioética & $\begin{array}{c}\text { Percepção de } \\
\text { profissionais da saúde } \\
\text { sobre abortamento legal. }\end{array}$ & $\begin{array}{l}\text { da Rocha } \\
\text { WB, et al. }\end{array}$ & $\begin{array}{l}\text { Identificar o conhecimento e a percepção dos } \\
\text { aspectos éticos envolvidos no Programa de } \\
\text { Interrupção Gestacional Prevista em Lei, por parte } \\
\text { de profissionais de saúde do Hospital Materno } \\
\text { Infantil de Brasília que não lidam diretamente com } \\
\text { os serviços de abortamento legal dessa } \\
\text { instituição. }\end{array}$ & $\begin{array}{l}\text { exploratório- } \\
\text { descritivo }\end{array}$ & $\begin{array}{l}\text { Quantiquali- } \\
\text { tativo }\end{array}$ & 2015 \\
\hline 6 & SCIELO & $\begin{array}{l}\text { Revista Brasileira } \\
\text { Ginecologia e } \\
\text { Obstetrícia. }\end{array}$ & $\begin{array}{c}\text { Influência da percepção } \\
\text { dos profissionais quanto } \\
\text { ao aborto provocado na } \\
\text { atenção à saúde da } \\
\text { mulher. }\end{array}$ & $\begin{array}{l}\text { Benute } \\
\text { GRG, et al. }\end{array}$ & $\begin{array}{c}\text { Identificar o conhecimento e a percepção dos } \\
\text { profissionais da saúde em relação à legislação } \\
\text { brasileira } \\
\text { sobre o aborto provocado. }\end{array}$ & $\begin{array}{l}\text { prospectivo e } \\
\text { transversal. }\end{array}$ & qualitativo & 2012 \\
\hline 7 & SCIELO & $\begin{array}{l}\text { Texto \& Contexto } \\
\text { - Enfermagem }\end{array}$ & $\begin{array}{c}\text { Percepções da } \\
\text { enfermagem sobre } \\
\text { gestão e cuidado no } \\
\text { abortamento: estudo } \\
\text { qualitativo. } \\
\end{array}$ & $\begin{array}{l}\text { Strefling ISS, } \\
\text { et al. }\end{array}$ & $\begin{array}{l}\text { Descrever a gestão e realização do cuidado às } \\
\text { mulheres hospitalizadas por abortamento sob a } \\
\text { perspectiva } \\
\text { dos profissionais de enfermagem. }\end{array}$ & $\begin{array}{l}\text { Exploratório- } \\
\text { descritivo }\end{array}$ & qualitativo & 2015 \\
\hline
\end{tabular}

Fonte: Andrade JS, et al., 2020.

REAS / EJCH | Vol.12(10) | e557 | DOI: https://doi.org/10.25248/reas.e557.2020 Página 5 de 9 


\section{DISCUSSÃO}

No contexto mais amplo, em grande parte dos autores percebe-se uma dualidade em relação a essa percepção da mulher na situação de abortamento, onde os mesmos revelam que o processo de cuidar de pacientes em situação em abortamento por si só muito limitado, sem que o cuidado integral seja colocado em prática, fato que contribui nos incrementos da razão de mortalidade materna no país.

Os estudos mostram que a percepção negativa dos profissionais sobre o abortamento e a mulher nesta situação, tem sido o fator mais importante para a precarização dos serviços de atenção à saúde sexual e reprodutiva. Os fatores envolvidos nesta percepção dos profissionais estão ligados diretamente à forma como são criados e adquirem o capital cultural dentro da sociedade (AQUINO EML, 2003 e MENEZES G, AQUINO EML, 2009).

Segundo Ayres $\mathrm{R}$, et al. (2018) os valores pessoais dos profissionais da equipe de enfermagem estão sendo colocado na pratica profissional durante a assistência. Rocha WB, et al. (2015) enfatiza que as dificuldades encontradas estão relacionadas por diversos fatores, entre eles a ignorância dos profissionais envolvidos na atenção ao abortamento sobre a legislação que rege tal prática, bem como, as representações sociais de cada profissional, muito fortemente permeada por suas crenças religiosas e a uma concepção de ética que criminaliza o aborto em qualquer circunstância e, portanto, acabam levando tais concepções para dentro de sua atividade laboral.

Segundo Silva JPL e Araújo MZ (2011), relacionam que os profissionais de enfermagem na pratica assistencial deixam influenciar por suas convicções pessoais que resulta na negligencia da atenção à saúde as mulheres em situação de abortamento. Segundo Gesteira SMA, et al. (2008) ao analisar a percepção de profissionais de enfermagem de uma maternidade pública de Salvador (BA) sobre a assistência prestada à mulher em processo de aborto provocado, constatou-se que esses profissionais entendem o aborto como crime e que a assistência prestada é discriminatória, mesmo nos casos de aborto previstos em lei.

Para Benute GRG, et al. (2011) e Strefing IS, et al. (2015) é notório o comportamento preconceituoso e a falta de diálogo entre os profissionais de enfermagem com as pacientes em processo de abortamento, que tende a interferir na prestação de uma assistência humanizada, tornando-a pautada no julgamento, na punição, na raiva e na discriminação segundo os valores que estes profissionais trazem.

Além disso, para que se tenha uma assistência de qualidade contemplando todos os aspectos envolvidos com mulheres em situação de abortamento, é preciso que os profissionais sejam conhecedores da legislação acerca do aborto que é contemplada no art. 128 do Código Penal, que destacam duas hipóteses onde o aborto não é punível, desde que praticado nos âmbitos legais e com profissionais qualificados, sendo estas: 1) se não houver outro meio de salvar a vida da gestante e, 2) se a gravidez resulta de estupro e o aborto é precedido do consentimento da gestante. Segundo Rocha WB, et al. (2015) relata que as ações da equipe de enfermagem que tentam interferir no processo de aborto julgam a manterem as gestações, não por caráter legal, e sim por aspectos religiosos.

Como consequência dessa visão permeada por conviç̧ões religiosas e conceitos pré-existentes tem-se um cuidado em saúde e em enfermagem que viola o direito das mulheres ao invés de acolhê-las. Estudo brasileiro que coletou história de mulheres assistidas em um hospital devido abortamento provocado evidenciou que o cuidado a estas mulheres foi feito sob forte violência dos direitos humanos destas, especialmente quando as pacientes confessaram que tinha sido aborto provocado.

Entre os maus-tratos e descriminações relatados, destacou-se a ameaça de denúncia do fato às autoridades policiais; demora no estabelecimento de medidas para o controle da dor; prolongamento da espera para a realização do procedimento de esvaziamento uterino e, internação, após o procedimento de curetagem uterina, no mesmo ambiente que as puérperas de parto normal e cesariana. Estes dados mostram que os profissionais acabam por serem protagonistas de grave violação dos direitos das mulheres nesta situação, fazendo com que acolhimento e atendimento de suas necessidades não se consolidem no cotidiano assistencial (MADEIRO AP e RUFINO AC, 2017). 
Neste sentido, a violência pratica pelos profissionais de saúde e em especial os de enfermagem à mulher em situação de abortamento é um fenômeno impregnado no cotidiano assistencial destes profissionais e soma-se à falta de estrutura física e de protocolos institucionais de cuidados a este público, fato que se caracteriza também como violência institucional às mulheres, fazendo com que o exercício do direito à saúde fique profundamente comprometido. Estes episódios de violência atuam também como comprometedor do processo de trabalho do enfermeiro, uma vez que diante de tais fatos não é possível o estabelecimento das relações de vínculo e, portanto, do cuidado (MADEIRO AP e RUFINO AC, 2017).

Segundo Strefing IS, et al. (2009), que enfatiza que a equipe de enfermagem ao assistirem o processo de aborto, trazem consigo que essas mulheres cometeram crime perante a lei e são pecadoras perante aos fatores religiosos. Esse pensamento profissional acerca do processo de abortamento influencia no atendimento à saúde dessas mulheres fragilizadas. Neste sentido, Silva JPL e Araújo MZ (2011) sintetizam que a percepção da equipe de enfermagem é discriminatória e predominantemente técnico e, quando comparado no aspecto do atendimento humanizado, apresenta um cuidado com vinculo solidário, e não com um direito humano.

Salienta-se ainda que o acolhimento do profissional de enfermagem à mulher em situação de abortamento sofre interferência dos julgamentos e discriminações imputados à usuária. Neste sentido, Silva JPL e Araújo MZ (2011) e Strefling IS, et al. (2015) citam que na norma técnica a atenção humanizada ao abortamento refere-se a proposta de acolhimento e orientação clínica a usuária pois o profissional deverá estabelecer uma relação de confiança ao invés de julgar a paciente, deixando com a mesma a autonomia e a capacidade de tomada de decisão.

A legislação brasileira sobre a temática do abortamento garante que todas as mulheres nesta condição possam ser inseridas e cuidados no sistema de saúde público do país. Assim, é urgente que todos os atores envolvidos no atendimento a estas usuárias possam ter formação necessária para prestar um cuidado integral e humanizado, sem que suas percepções sobre o aborto influenciem no processo.

Segundo Yam EA, et al. (2006) a conduta na assistência da equipe de enfermagem desempenha um papel primordial no processo de abortamento, seja admitindo a paciente em processo de abortamento (nos casos de legalidade, ou não); seja negando-se a fazê-lo com base nos recursos éticos e morais. Outrossim, os autores propõem a importância de prestar um atendimento digno e humano, independentemente da etiologia do aborto, e apontam alguns aspectos humanizados como a escuta, o apoio psicológico e o provimento e um ambiente terapêutico exclusivo e separado das demais pacientes, a fim de qualificar 0 atendimento.

Segundo Gesteira SMA, et al. (2008) relata ainda a existência de uma percepção por parte dos enfermeiros, onde as mulheres que engravidam sem planejar são ditas como irresponsáveis, tal discurso traz à tona questões éticas e morais e a negação do exercício dos direitos sexuais e reprodutivos das mulheres, que foi conquistando e vem sendo defendidas na sociedade através do movimento feminista e políticas governamentais, sem falar que traz à tona uma das causas de precarização na prestação dos cuidados.

Todavia Martins AC, et al. (2018) contextualiza que o enfermeiro apesar de estar inserido no contexto saúde da mulher, entende que esta não é a única responsável pelo planejamento reprodutivo do casal e neste sentido, trazendo de forma implícita a não responsabilização dos eventos relacionados ao ciclo gravídico como sendo somente dela.

Segundo Ayres R, et al. (2018) na equipe de enfermagem nem sempre se considera as diferenças de gêneros, sendo preciso reconhecer que homens e mulheres de acordo com seus pensamentos éticos, legais e culturais, dispõem de referenciais diferentes para compreender e atuar frente ao processo de abortamento em virtudes de suas vivencias de gênero.

Por outro lado, novas evidências descrevem mudanças no modelo de formação dos profissionais de enfermagem no sentido de avançar as discussões sobre a mulher em situação de abortamento e o cuidado dispensado a elas. 
Em um estudo desenvolvido com acadêmicos de enfermagem de uma instituição de ensino superior no nordeste brasileiro, para verificar sua percepção sobre o cuidado à mulher diante do aborto provocado, os resultados mostraram que a maioria dos estudantes acreditam que a legalização do aborto no Brasil trará redução das taxas anuais. No tocante ao cuidado, todos os estudantes pesquisados afirmaram que é necessário que os profissionais de enfermagem possam estabelecer o acolhimento necessário para a criação do vínculo com a usuária e que o processo de trabalho em enfermagem deve objetivar a preservação da integridade da mulher, com atendimento de suas necessidades humanas básicas, afim de proporcionar o bem-estar da mesma (DUQUE NC, et al., 2020).

A mudança de paradigmas na concepção do cuidado de enfermagem no que diz respeito ao abortamento perpassa pela mudança dos aspectos atitudinais dos profissionais de enfermagem em formação. Neste sentido, nota-se que os estudantes de enfermagem compreendem que uma boa formação para a atenção às mulheres neste contexto específico deve ser caracterizada por uma imersão em conteúdos relacionados aos fatores sociais e culturais ligados ao abortamento, à legislação de estado e de enfermagem sobre o assunto, além dos aspectos que envolvam a anatomia e a fisiologia e os cuidados clínicos necessários (DUQUE NC, et al., 2020).

Também, é necessário que se compreenda que essas práxis negativadas sobre o cuidado à mulher neste contexto do aborto, seja espontâneo ou provocado é uma construção histórico-social que se confunde com a construção das identidades culturais e principalmente religiosas das populações nas diversas sociedades, nos diferentes contextos e temporalidades.

Ao longo do tempo as concepções sobre o aborto sofreram inúmeras influências de vertentes sociais, políticas, religiosas e jurídicas, com o surgimento e predomínio de duas correntes de pensamento sobre o tema: a concepcionista, que considerada a vida deste o momento da fecundação e a natalícia, que considera a vida humana somente após o parto (BASTOS PMB, 2017).

A partir destas duas concepções de vida, surgiram os conflitos em relação ao conceito do abortamento e sua prática e com a expansão da religião cristã houve uma consolidação da visão concepcionista a respeito do tema, fato que se fortaleceu com as revoluções francesa e industrial onde a difusão da ideia de aumento da população era benéfica em trazer extensa mão de obra barata, além de consolidar a máxima de nação populosa como nação forte. Estas concepções são profundamente arraigadas na sociedade, e que até os dias atuais constituem a base para as concepções do abortamento entre os profissionais que se traduzem em uma assistência permeada pelo preconceito e discriminação (BASTOS PMB, 2017).

Por fim, Rocha WB, et al. (2015) mencionam o aprofundamento do conhecimento sobre o abortamento, a mulher em situação de abortamento e suas várias interfaces dentro dos contextos nos quais o mesmo se perpassa pode gerar mudanças comportamentais e atitudinais sobre a problemática em tela, fazendo com que os cuidados dos profissionais de saúde, e mais especificamente os de enfermagem, possam acontecer para promover a dignidade das mulheres que vivenciam esta situação.

\section{CONSIDERAÇÕES FINAIS}

Ao final desse estudo percebe-se que a percepção evidenciada dos enfermeiros sobre as mulheres em situação de abortamento é extremamente negativa por questões da falta de ética profissional, onde a atitude e valores sociais visam questões religiosas, culturais, econômicas e sociais que influenciam muito negativamente na assistência prestada a essas pacientes, marginalizando-as. É notório, a partir das análises feitas, que o preconceito, onde é um fator que influencia fortemente no mau atendimento no contexto de atenção às mulheres em situação de abortamento no Brasil. Vale frisar ainda que há uma deficiência de publicações recentes voltadas à temática abordada, mostrando fragilidades inclusive acadêmicas sobre o assunto. 


\section{REFERÊNCIAS}

1. AQUINO EML, et al. Adolescência e reprodução no Brasil: a heterogeneidade dos perfis sociais. Cadernos de Saúde Pública, 2003; 19(Sup. 2):S377-S388.

2. AYRES R, et al. A contextualização do aborto sob a ótica do enfermeiro. Nursing (Säo Paulo), $2018 ; 21$ (244):23342337.

3. BASTOS PMB. A percepção do aborto ao longo da história e a construção do seu conceito nos dias atuais. Revista Unitas, 2017; 5(2): 55-70.

4. BENUTE GRG, et al. Influência da percepção dos profissionais quanto ao aborto provocado na atenção à saúde da mulher. Revista Brasileira de Ginecologia e Obstetrícia, 2012; 34 (2): 69-73.

5. BRASIL. Ministério da saúde. Manual de Controle de Doenças Sexualmente Transmissíveis. 3. ed., Brasília, 1999.

6. BRASIL. Ministério da Saúde. Secretaria de Atenção à Saúde. Departamento de Ações Programáticas Estratégicas. Área Técnica de Saúde da Mulher. Atenção humanizada ao abortamento: norma técnica. Brasília, 2005.

7. BRASIL. Ministério da saúde. Secretaria de atenção à saúde. Departamento de ações Programáticas estratégicas. Atenção humanizada ao abortamento: Norma técnica / ministério da saúde, secretaria de atenção à saúde, Área técnica de saúde da mulher. - 2. ed. - Brasília, 2011.

8. BRASIL. Ministério da Saúde. Secretaria de Ciência, Tecnologia e Insumos Estratégicos. Departamento de Ciência e Tecnologia. Aborto e saúde pública no Brasil: 20 anos / Ministério da Saúde, Secretaria de Ciência, Tecnologia e Insumos Estratégicos, Departamento de Ciência e Tecnologia. - Brasília: Ministério da Saúde, 2009.

9. DOMINGOS SRF, MERIGHI MAB. O aborto como causa de mortalidade materna: um pensar para o cuidado de enfermagem. Escola Anna Nery Revista de Enfermagem, 2010; 14(1): 177-181.

10. DUQUE NC, et al. Perception of nursing academics in connection with provoced abortion. Brazilian Applied Science Review, 2020; 4(3): 1890-1900.

11. GALLI B. Dossiê sobre aborto inseguro para advocacy: o impacto da ilegalidade do abortamento na saúde das mulheres e na qualidade da atenção à saúde reprodutiva no Estado do Rio de Janeiro. Recife: Grupo Curumin/Rio de Janeiro: Ipas Brasil, 2010.

12. GESTEIRA SMA, et al. Assistência à mulher em processo de abortamento provocado: discurso de profissionais de enfermagem. Acta Paulista de Enfermagem, 2008; 21(3):449-53.

13. MADEIRO AP, RUFINO AC. Maus-tratos e discriminação na assistência ao aborto provocado: a percepção das mulheres em Teresina, Piauí, Brasil. Ciência \& Saúde Coletiva, 2017; 22(8): 2771-2780.

14. MARTINS AC, et al. Paternidade na gestação e parturição: uma revisão integrativa. Revista Família, Ciclos de Vida e Saúde no Contexto Social, 2018; 6 (3): 485-493.

15. MENDES KDS, et al. Revisão Integrativa: método de pesquisa para a incorporação de evidências na saúde e na enfermagem. Texto e Contexto Enfermagem, 2008; 17(4): 758-64.

16. MENEZES G, AQUINO EML. Pesquisa sobre o aborto no Brasil: avanços e desafios para o campo da saúde coletiva. Cadernos de Saúde Pública, 2009; 25 (Sup 2):S193-S204.

17. ROCHA WB, et al. Percepção de profissionais da saúde sobre abortamento legal. Revista Bioética, $2015 ; 23$ (2): 387 99.

18. SILVA JPL, ARAÚJO MZ. Olhar reflexivo sobre o aborto na visão da enfermagem a partir de uma leitura de gênero. Revista Brasileira de Ciências da Saúde, 2011; 14(4):19-24.

19. STREFLING IS, et al. Percepções da enfermagem sobre gestão e cuidado no abortamento: estudo qualitativo. Texto \& Contexto - Enfermagem, 2015; 24 (3): 784-791.

20. YAM EA, et al. Pesquisa de opinião sobre aborto na América Latina e no Caribe: uma revisão da literatura. Estudos em Planejamento Familiar, 2006; 37 (4): 225-240. 